IJMMS 31:4 (2002) 193-199

PII. S0161171202013066

http://ijmms.hindawi.com

(c) Hindawi Publishing Corp.

\title{
ASPECTS OF COMPLETE UNIFORM SPREADS IN FRAMES
}

\author{
HLENGANI JAMES SIWEYA
}

Received 1 May 2001 and in revised form 16 January 2002

We give a new notion of a complete uniform spread in terms of a relative kind of uniform local connectedness. Properties of this type of (uniform) connectedness are discussed. It is also shown that our concept of complete spread is equivalent to that of Hunt (1982).

2000 Mathematics Subject Classification: 54D15, 54E15.

1. Introduction. The concept of a complete uniform spread together with its completion was introduced into the category $\mathbb{U}$ nif of uniform spaces and uniformly continuous functions by Hunt [6] (see also [7]). In [11], we introduced these concepts into the category $U \mathbf{n i} \Vdash r r m$ of uniform frames and uniform homomorphisms.

Section 2 is devoted to properties of uniform local connectedness with respect to along. We also show how the Banaschewski-Pultr uniform frame completion $C L$ of a uniform frame $L$ can be used to obtain point-free analogies of some well-known topological results. Section 3 is devoted to complete spreads. Hunt [8] defines a uniform spread $f:(X, \mathcal{U}) \rightarrow(Y, \mathscr{R})$ to be complete if $(X, \mathcal{U})$ is a complete uniform space where $\checkmark$ is a spread uniformity generated by $f$. We give a new definition of a complete uniform spread in terms of the concept of uniform local connectedness with respect to along. It is then shown that such a definition is equivalent to that of Hunt (1982).

We assume familiarity with general knowledge of frames, especially [9] and uniform frames according to $[3,4,5]$. Briefly, a cover of a frame $L$ is a subset $A \subseteq L$ such that $\bigvee A=e$, the top element of $L$. A cover $U$ of $L$ is said to refine a cover $V$ of $L$ if for each $x \in U$ there is $y \in V$ such that $x \leq y$. We write $U \leq V$. Given a cover $A$ of $L$ and $x \in L$, we define the $A$-star of $x$ to be the element

$$
A x=\bigvee\{y \in A \mid x \wedge y \neq 0\} .
$$

A cover $A$ of $L$ star-refines another cover $B$ (denoted $A \leq^{*} B$ ) whenever $\{A x \mid x \in$ $A\} \leq B$. Let $\operatorname{Cov}(L)=\{A \leq L \mid A$ is a cover of $L\}$. A collection $\mathfrak{U} L \subseteq \operatorname{Cov}(L)$ is called a pre-uniformity on $L$ if

(1) $A \wedge B \in \mathfrak{U} L$ for all $A, B \in \mathfrak{U} L$, where $A \wedge B=\{x \wedge y \mid x \in A, y \in B\}$;

(2) $B \geq A \in \mathfrak{U} L$ implies $B \in \mathfrak{U} L$;

(3) for every $A \in \mathfrak{U} L$ there is $B \in \mathfrak{U} L$ such that $B \leq^{*} A$.

A pre-uniformity $\mathfrak{U} L$ is called a uniformity on $L$ provided that it satisfies the compatibility condition: for each $a \in L$,

$$
a=\bigvee\{a / C \mid C \in \mathfrak{U} L\},
$$

where $a / C=\bigvee\{x \in L \mid C x \leq a\}$. 
Our notions of connectedness are those of Baboolal and Banaschewski [2]. Given a frame $L$, we say that an element $z \in L$ is connected if whenever $z=x \vee y$ with $x \wedge y=0$, then $x=0$ or $y=0$, and a cover of $L$ is connected provided that its elements are connected. The frame $L$ is connected whenever its top element $e$ is connected, and it is locally connected if

$$
x=\bigvee\{y \in L \mid y \text { is connected, } y \leq x\},
$$

for each $x \in L$. An element $x \in L$ is a component of an element $y \in L$ if $x \leq y$ is maximally connected. Throughout the paper, we use the shorter $x \leq_{c} y$ to mean that $x$ is a component of $y$. The following concept was introduced in [11].

DEFinition 1.1. A frame homomorphism $h: L \rightarrow M$ between locally connected frames is called a localic spread if $\bigcup\left\{S_{u} \mid u \in L\right\}$ is a basis for $M$ where, for each $u \in L$,

$$
S_{u}=\left\{x \in M \mid x \leq_{c} h(u)\right\} .
$$

Now, consider a localic spread $h: L \rightarrow M$ which is onto with $L$ carrying the fine uniformity $\mathfrak{U}_{F} L$. (The fine uniformity on a uniform frame $L$ is the uniformity generated by all normal covers, that is, those covers $A$ of $L$ such that $A=A_{1}$ in some sequence $\left(A_{n}\right)_{n}$ of covers of $L$ such that $A_{n+1} \leq^{*} A_{n}$ for all $n=1,2,3, \ldots$ ) For each $U \in \mathfrak{U}_{F} L$, let

$$
T_{U}=\left\{z \in M \mid z \leq_{c} h(u) \text {, for some } u \in U\right\} .
$$

LEMMA 1.2. The collection

$$
\left\{T_{U} \mid U \in \mathfrak{U}_{F} L\right\}
$$

is a basis for a uniformity on $M$.

Proof. The proof (which is found in [12]) is a consequence of Baboolal [1, Theorem 3.1].

DEFinition 1.3. The uniformity generated by the basis of the lemma is called the spread uniformity (on $M$ ) and $h$ is called a uniform spread. Hereafter, the spread uniformity on $M$ is denoted by $\mathfrak{L}^{h} M$.

Recall [2] that a uniform frame $L$ is said to be uniformly locally connected if each cover $V$ of $L$ is refined by a cover $U$ each of whose elements is connected. So, in the definition of a uniform spread, the underlying uniform frames are uniformly locally connected. Moreover, a uniform spread is a uniform homomorphism: for, suppose $h[A] \in \mathfrak{U}_{w} M$ and take $h(x) \in h[A]$ for $A \in \mathfrak{U}_{F} L$ where $\mathfrak{U}_{w} M$ is the weak uniformity on $M$. Then $T_{A} \leq h[A]$ : if $y \in T_{A}$, then $y \leq h\left(u_{y}\right)$ for some $u_{y} \in A$ so that $T_{A} \leq h[A]$. Since $T_{A} \in \mathfrak{U}^{h} M$, we must have $h[A] \in \mathfrak{U}^{h} M$.

2. Uniform local connectedness with respect to along. We introduce the notion of uniform local connectedness with respect to along for uniform frames and prove that for dense surjections, this concept coincides with uniform local connectedness introduced by Baboolal in [1]. The significance of this concept is given in Section 3 where our approach to complete uniform spreads has a bearing on uniform local connectedness with respect to along. 
DEFINITION 2.1. Let $h:(L, \mathfrak{U} L) \rightarrow(M, \mathfrak{U} M)$ be a surjective homomorphism. We say that $M$ is uniformly locally connected with respect to $L$ along $h$ if there is a basis $\mathscr{B}$ for the uniformity $\mathfrak{U} L$ such that each cover $h[B]$ is connected for $B \in \mathscr{B}$, where

$$
h[B]=\{h(b) \mid b \in B\} .
$$

Proposition 2.2. Let $h: L \rightarrow M$ be a surjective homomorphism. If $M$ is uniformly locally connected with respect to $L$ along $h$, then $M$ is uniformly locally connected. So, in particular, if $M$ is uniformly locally connected with respect to $L$ along $h$, then $L$ is uniformly locally connected.

Proof. The second statement is a consequence of a result of Baboolal [1]. Assume that $h: L \rightarrow M$ is a surjective homomorphism and that $M$ is uniformly locally connected with respect to $L$ along $h$. Then given $A \in \mathfrak{U} M$, we have $h[B] \leq A$ for some $B \in \mathfrak{U} L$. There is a basis $\mathscr{B}$ for $\mathfrak{U} L$ for which $h[U]$ is connected, for each $U \in \mathscr{B}$. Moreover, there is a uniform cover $C \in \mathscr{B}$ such that $C \leq B$. Then $h[C] \leq h[B] \leq A$. Since each $h[C]$ is connected for $C \in \mathscr{B}, M$ must be uniformly locally connected.

Proposition 2.3. Under the hypothesis of Proposition 2.2, if $h: L \rightarrow M$ is a dense surjection, then $M$ is uniformly locally connected if and only if $M$ is uniformly locally connected with respect to $L$ along $h$.

Proof. Suppose that $M$ is uniformly locally connected. Then by hypothesis, $\mathfrak{U} M$ is generated by all $C \in \mathfrak{U} M$ such that $C$ is a connected cover. We claim that the collection

$$
\left\{h_{*}[C] \mid C \in \mathfrak{U} M, C \text { connected }\right\}
$$

is a basis for $\mathcal{U} L$ and that $h \circ h_{*}[C]=C$

(i) we first show that $h_{*}[C] \in \mathfrak{U} L$ : let $C \in \mathfrak{U} M$. Then there exists a uniform cover $A \in \mathfrak{U} L$ such that $h[A] \leq C$ so that $A \leq h_{*}[C]$;

(ii) given $A \in \mathfrak{U} L$, pick $B \in \mathfrak{U} L$ such that $B \leq^{*} A$. Then $h[B] \in \mathfrak{U} M$. We show that $h_{*} \circ h[B] \leq A$ : pick any $b \in B$. Then $B b \leq a$, for some $a \in A$ which implies that $b^{*} \vee a=e$. Now,

$$
h\left(\left(h_{*} \circ h(b)\right) \wedge b^{*}\right)=h(b) \wedge h\left(b^{*}\right)=0 .
$$

Since $h$ is dense $\left(h_{*} \circ h(b)\right) \wedge b^{*}=0$. Now, since $b^{*} \vee a=e$ it follows that $h_{*} \circ h(b) \leq$ $a$ which gives $h_{*} \circ h[B] \leq A$. Therefore, since $M$ is uniformly locally connected, there is a connected cover $C \in \mathfrak{U} M$ such that $C \leq h[B]$ so that $h_{*}[C] \leq h_{*} \circ h[B]$. Thus, $h_{*}[C] \leq A$.

In [3], Banaschewski and Pultr described the uniform (frame) completion of a uniform frame $L$ as a certain quotient of the down-set $D L$ of $L$. (We refer to this completion as the Banaschewski-Pultr uniform frame completion to distinguish it from Isbell's [9] and Kř́źž's [10].) Using such a completion, we now give point-free versions of some well-known topological results. The notation used in the applications below evolves from the Banaschewski-Pultr uniform frame completion.

The following result (proved in [12]) is a point-free version of the well-known topological fact that in $\mathbb{U}$ nif every uniformly continuous function on a dense subspace of 
a uniform space into a complete uniform space has a unique uniformly continuous extension (see [5]).

Proposition 2.4. Let $(L, \mathfrak{U L} L)$ be a complete uniform frame, let $h:(L, \mathfrak{U} L) \rightarrow(M, \mathfrak{U} L)$ be a uniform frame homomorphism, and let $\rho:(N, \mathfrak{U N}) \rightarrow(M, \mathfrak{U} M)$ be a dense surjection. Then there is a unique uniform frame homomorphism $g: L \rightarrow N$ such that $\rho \circ g=h$

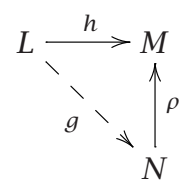

COROLlaRY 2.5. Given a dense surjection $f_{i}: M_{i} \rightarrow L_{i}$ from a complete uniform frame to a uniform frame for $i=1,2$, if $h: L_{1} \rightarrow L_{2}$ is an isomorphism, then there exists a unique isomorphism $g: M_{1} \rightarrow M_{2}$ such that $f_{2} \circ g=h \circ f_{1}$

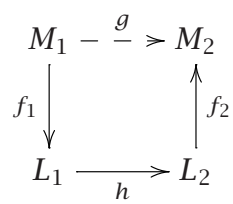

Proof. By Proposition 2.4, there exist unique uniform homomorphisms $g: M_{1} \rightarrow$ $M_{2}$ and $k: M_{2} \rightarrow M_{1}$ such that $f_{2} \circ g=h \circ f_{1}$ and $f_{1} \circ k=h^{-1} \circ f_{2}$
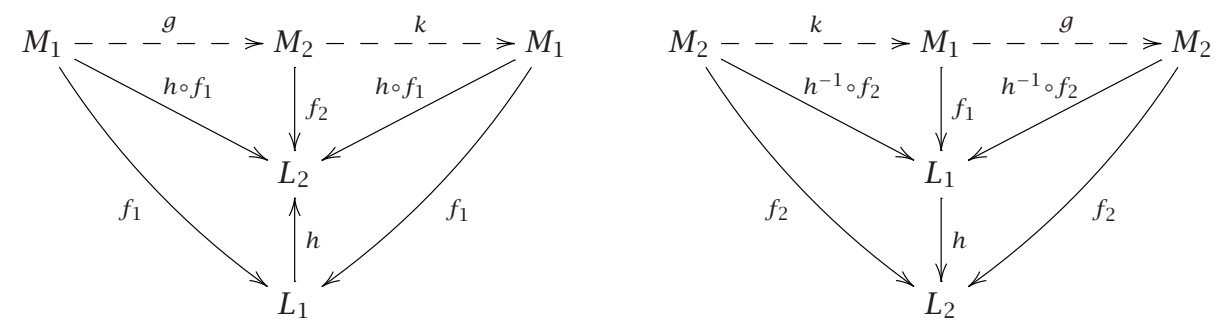

Since $f_{1}$ and $f_{2}$ are monic in $\mathbb{R} \mathbf{e g} \llbracket \mathbf{r m}$, it follows from the commutativity of the diagrams that $g \circ k=\mathrm{id}_{M_{2}}$ and $k \circ g=\operatorname{id}_{M_{1}}$.

3. Complete uniform spreads. Hunt [8] defines a uniform spread $(X, f, Z)$ to be complete if $(X, \mathfrak{l})$ is a complete uniform space, where $\mathfrak{U}$ is the spread uniformity generated by $f$ on $X$. The uniform spreads $(L, h, M)$ dealt with here are those for which the "base" uniform frame $L$ is complete with respect to some uniformity on $L$. (Such frames are called frame complete frames [11].)

Our notion of a complete spread is in terms of uniform local connectedness with respect to along. We will show that our definition is equivalent to that of Hunt. In [11], we have shown that our notion of completeness for spreads can be used to obtain point-free versions of Hunt's uniform properties spreads. 
DEFinition 3.1. An extension of a uniform spread $(L, h, M)$ is a uniform spread $(L, g, N)$ together with a dense surjection $f: N \rightarrow M$ where $M$ is uniformly locally connected with respect to $N$ along $f$ such that $f \circ g=h$. A uniform spread $(L, h, M)$ is said to be complete if, whenever $(L, g, N)$ is a uniform spread and $f: N \rightarrow M$ is a dense surjection with $M$ uniformly locally connected with respect to $N$ along $f$ such that the triangle

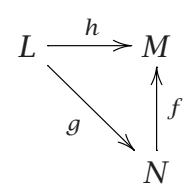

commutes, then $f$ is an isomorphism.

Proposition 3.2. The completeness of a uniform spread is frame invariant under spread isomorphisms.

Proof. Suppose that $(L, h, M)$ and $(H, g, K)$ are isomorphic uniform spreads with a spread isomorphism $(f, k):(L, h, M) \rightarrow(H, g, K)$ with $(H, g, K)$ complete. To see that $(L, h, M)$ is also complete, take a uniform spread $(L, S, N)$ and a dense surjection $t: N \rightarrow M$ with $M$ uniformly locally connected with respect to $N$ along $t$ such that $h=t \circ s$

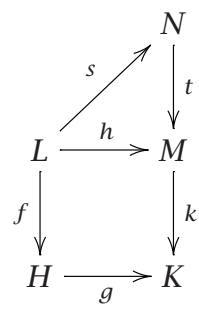

It is easily shown that $t$ is an isomorphism.

LEMMA 3.3. Let $h:(L, \mathfrak{U} L) \rightarrow\left(M, \mathfrak{U}^{h} M\right)$ be a uniform spread. If $g:(L, \mathfrak{U} L) \rightarrow\left(N, \mathfrak{U}^{g} N\right)$ is a uniform spread and $f:(N, \mathfrak{U N}) \rightarrow\left(M, \mathfrak{U}^{h} M\right)$ is a dense surjection such that $h=$ $f \circ g$, then $\mathfrak{U} N \subseteq \mathfrak{U}^{g} N$ (i.e., $f:\left(N, \mathfrak{l}^{g} N\right) \rightarrow\left(M, \mathfrak{U}^{h} M\right)$ is also a dense surjection).

Proof. Since $f$ is dense, the denseness of the desired homomorphism is immediate. To show that $\mathfrak{U} N \subseteq \mathfrak{U}^{g} N$, we need only to show that for each $A \in \mathfrak{U} N$ there exists $T_{W} \in \mathfrak{U}^{g} N$ satisfying $T_{W} \leq A$.

Given $A \in \mathfrak{U} N$, since $f$ is a surjection, there exists $T_{V} \in \mathfrak{U}^{h} M$ such that $f_{*}\left[T_{V}\right] \leq A$ with $V \in \mathfrak{U}_{F} L$. So, it is enough to find $T_{W} \in \mathfrak{U}^{g} N$ satisfying $T_{W} \leq f_{*}\left[T_{V}\right]$. Now there is $T_{W} \in \mathfrak{U}^{g} N$ such that $T_{W} \leq \mathfrak{g}[V]$ for $W \in \mathfrak{U}_{F} L$.

We claim that $T_{W} \leq f_{*}\left[T_{V}\right]$ : take $y \in T_{W}$. Then $y \leq_{c} g(w)$ for some $w \in W$. But then $y \leq g(v)$ for some $v \in V$. Now we have $f(y) \leq f \circ g(v)=h(v)$. Since $M$ is locally connected (being uniformly locally connected [2]), we may (and do) assume that

$$
h(v)=\bigvee a \quad\left(a \leq_{c} h(v)\right) .
$$


Now recall that for a dense onto frame homomorphism $f: L \rightarrow M$ with $M$ locally connected with respect to $L$ along $f$, the right adjoint $f_{*}$ preserves pairwise disjoint joins [11]. So, in particular, we have

$$
y \leq f_{*} \circ f(y) \leq \bigvee f_{*}(a) \quad\left(a \leq_{c} h(v)\right) .
$$

Now since the $f_{*}(a)$ are pairwise disjoint and $y$ is connected it follows that $y \leq$ $f_{*}(a)$, for some $a \leq_{c} h(v)$-which proves that $T_{W} \leq f_{*}\left[T_{V}\right]$.

Proposition 3.4. A uniform spread $h:(L, \mathfrak{U} L) \rightarrow\left(M, \mathfrak{U}^{h} M\right)$ is a complete uniform spread if and only if $\left(M, \mathfrak{U}^{h} M\right)$ is a complete uniform frame; that is, $h$ is complete in the sense of Definition 3.1 if and only if it is complete in the sense of Hunt.

Proof. $(\Longrightarrow)$ Suppose that $h:(L, \mathfrak{U} L) \rightarrow\left(M, \mathfrak{U}^{h} M\right)$ is complete and let $p:(N, \mathfrak{U} N) \rightarrow$ $\left(M, \mathfrak{U}^{h} M\right)$ be a dense surjection. Since $L$ is complete, it follows that $h$ extends to a uniform spread, say $g: L \rightarrow\left(N, \mathfrak{U}^{g} N\right)$ by the construction in Proposition 2.4-such an extension is precisely the composition

$$
g=\gamma_{N} \circ(C p)^{-1} \circ C h \circ \gamma_{L}^{-1},
$$

and (trivially) $p \circ g=h$.

Since $p$ is a dense surjection, it follows (Lemma 3.3) that $p:\left(N, \mathfrak{U}^{g} N\right) \rightarrow\left(M, \mathfrak{U}^{h} M\right)$ is also a dense surjection. Now because $M$ is uniformly locally connected, it follows that $M$ is uniformly locally connected with respect to $N$ along $p$ (Proposition 2.3). Therefore, since $h$ is complete and since $p \circ g=h$ with $g$ being a uniform spread, it is implied that $p$ is an isomorphism, thus, $M$ is a complete uniform frame.

ACKNowledgments. This paper was presented at the Pan Africanist Congress of Mathematicians Conference (January 2000) at the University of Western Cape. I am grateful to the University of the North for financial assistance, which enabled me to attend the conference.

\section{REFERENCES}

[1] D. Baboolal, Local connectedness made uniform, Appl. Categ. Structures 8 (2000), no. 1-2, 377-390.

[2] D. Baboolal and B. Banaschewski, Compactification and local connectedness of frames, J. Pure Appl. Algebra 70 (1991), no. 1-2, 3-16.

[3] B. Banaschewski and A. Pultr, Samuel compactification and completion of uniform frames, Math. Proc. Cambridge Philos. Soc. 108 (1990), no. 1, 63-78.

[4] _ Paracompactness revisited, Appl. Categ. Structures 1 (1993), no. 2, 181-190.

[5] D. Bushaw, Elements of General Topology, John Wiley \& Sons, New York, 1963.

[6] J. H. V. Hunt, Branched coverings as uniform completions of unbranched coverings (Résumé), Symposium on Algebraic Topology in Honor of José Adem, (Proceedings, Oaxtepec, Mor., Mexico, 1981) (S. Gilter, ed.), American Mathematical Society, Rhode Island, 1982, pp. 141-155.

[7]___ Branched Coverings as Uniform Completions of Unbranched Coverings (Résumé), Contemporary Mathematics, vol. 12, American Mathematical Society, 1982.

[8] _ The uniform properties of Fox's spreads, Bol. Soc. Mat. Mexicana (2) 34 (1989), no. 1-2, 11-21.

[9] J. R. Isbell, Atomless parts of spaces, Math. Scand. 31 (1972), 5-32. 
[10] I. Kříž, A direct description of uniform completion in locales and a characterization of LT-groups, Cahiers Topologie Géom. Différentielle Catég. 27 (1986), no. 1, 19-34.

[11] H. J. Siweya, Spreads in locales and uniform locales, Ph.D. thesis, University of DurbanWestville, 2000.

[12] _ Spread uniformities and uniform spreads, Quaestiones Math. 24 (2001), no. 2, 157-163.

Hlengani James Siweya: Department of Mathematics, University of the North, Private BAG X1106, Sovenga 0727, SOUTH AFricA

E-mail address: siweyah@unorth.ac.za 


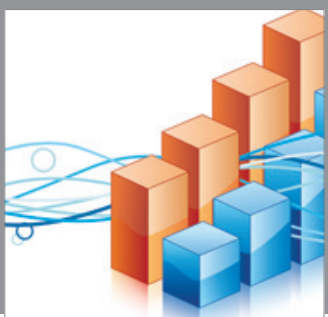

Advances in

Operations Research

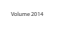

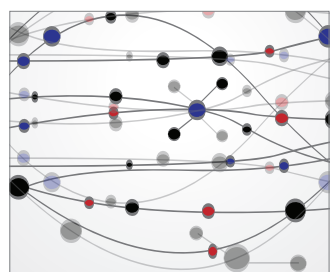

\section{The Scientific} World Journal
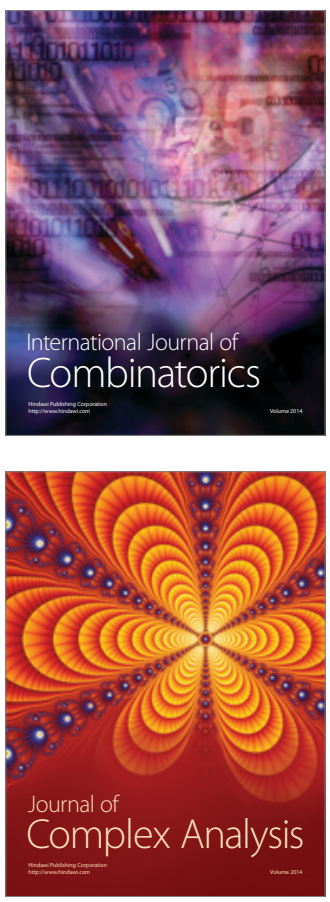

International Journal of

Mathematics and

Mathematical

Sciences
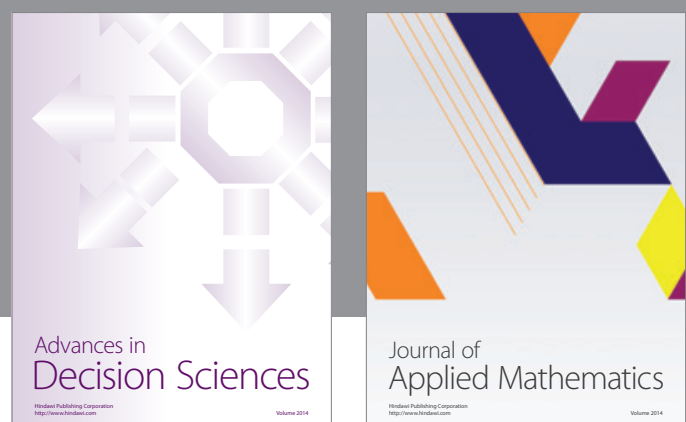

Journal of

Applied Mathematics
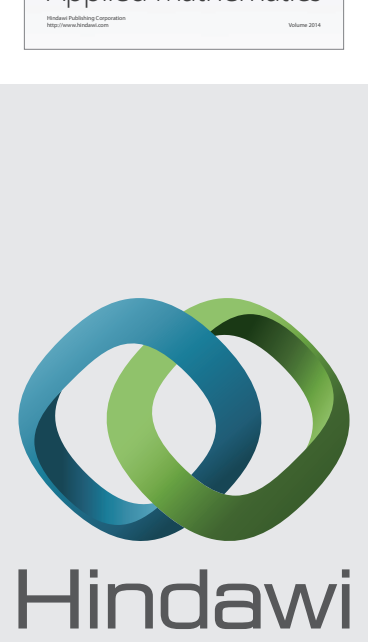

Submit your manuscripts at http://www.hindawi.com
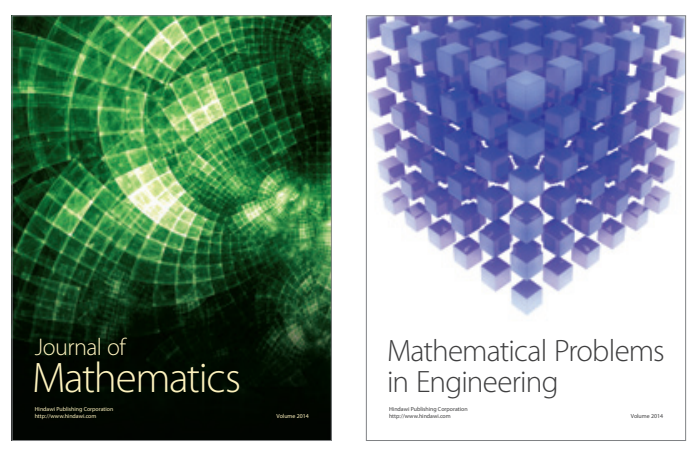

Mathematical Problems in Engineering
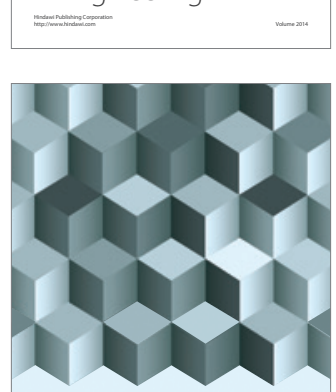

Journal of

Function Spaces
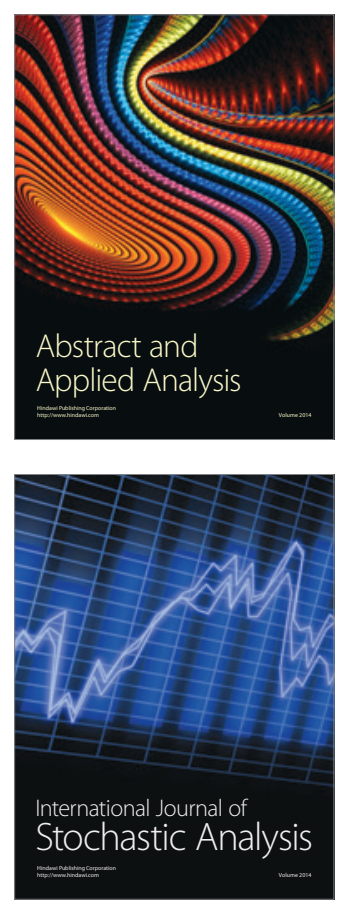

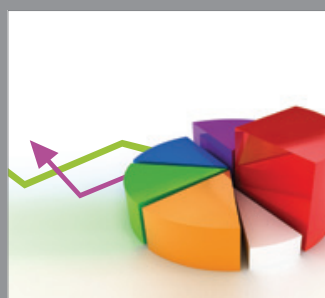

ournal of

Probability and Statistics

Promensencen
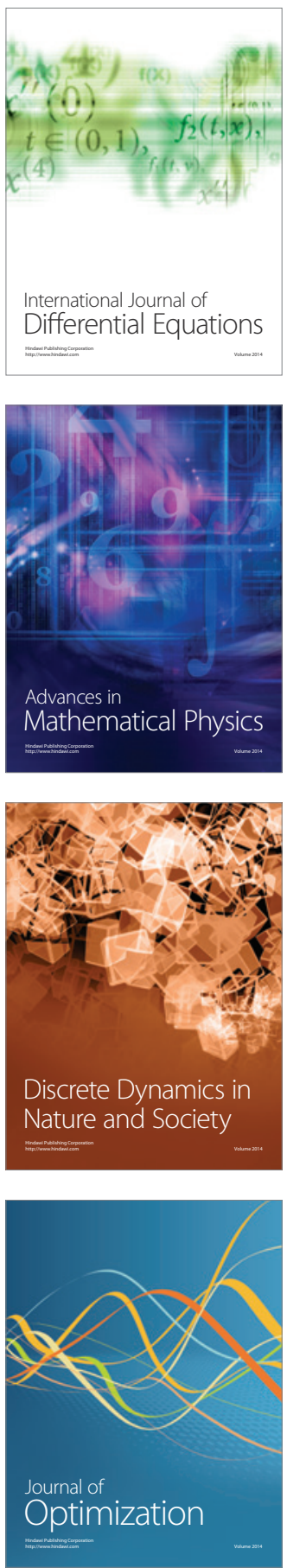